University of Nebraska - Lincoln

DigitalCommons@University of Nebraska - Lincoln

CSE Conference and Workshop Papers

Computer Science and Engineering, Department

2011

\title{
A Real-time RFID Localization Experiment Using Propagation Models
}

Jason L. Brchan

University of Nebraska-Lincoln, jbrchan@huskers.unl.edu

Lianlin Zhao

University of Nebraska-Lincoln, Izhao@huskers.unl.edu

Jiaqing $\mathrm{Wu}$

University of Nebraska-Lincoln, wujiaqing@huskers.unl.edu

Follow this and additional works at: https://digitalcommons.unl.edu/cseconfwork

Part of the Computer Sciences Commons

Brchan, Jason L.; Zhao, Lianlin; and Wu, Jiaqing, "A Real-time RFID Localization Experiment Using Propagation Models" (2011). CSE Conference and Workshop Papers. 195.

https://digitalcommons.unl.edu/cseconfwork/195

This Article is brought to you for free and open access by the Computer Science and Engineering, Department of at DigitalCommons@University of Nebraska - Lincoln. It has been accepted for inclusion in CSE Conference and Workshop Papers by an authorized administrator of DigitalCommons@University of Nebraska - Lincoln. 


\section{A Real-time RFID Localization Experiment Using Propagation Models}

\author{
Jason L. Brchan \\ University of Nebraska - Lincoln \\ email: jbrchan@huskers.unl.edu
}

\author{
Lianlin Zhao \\ University of Nebraska - Lincoln \\ email: lzhao@huskers.unl.edu
}

\author{
Jiaqing $\mathrm{Wu}$ \\ University of Nebraska - Lincoln \\ email: wujiaqing@huskers.unl.edu
}

\author{
Robert E Williams, Ph.D. \\ University of Nebraska - Lincoln \\ email: rwilliams2@unl.edu
}

Lance C. Pérez, Ph.D.

University of Nebraska - Lincoln

email: lperez@unl.edu

\begin{abstract}
This paper introduces a real-time localization system (RTLS) using efficient multiple propagation models to compensate for the drawback of the received signal strength technique. The RTLS is implemented on an active RFID system and uses received signal strength measurements and reference tags for ranging. The RTLS is implemented purely in software that post processes the received signal strength data from the reader and does not require any additional hardware or any modifications to the RFID reader or tags. The proposed algorithm using multiple propagation models improves the performance of the RTLS. Two-dimensional localization results are given for a four-reader system covering a 4.5 by 5.5 meter room. The scenarios of both single tag and two tags for the tag object are developed. It has been proven that tag multiplicity, two tags for the target object, improves the performance of the system by reducing inaccurate received signal strength measurements due to poor tag orientation. Experimental results show that the proposed system achieves a localization accuracy within 1 meter in over 50 percent of the experiments and outperforms other comparable systems. Currently developed three-dimensional space extension research is discussed and results are presented.
\end{abstract}

Index Terms-RFID, RTLS, localization, triangulation, RSSI, propagation modeling.

\section{INTRODUCTION}

RFID represents a way of identifying objects or people using radio waves [1]. The simplicity of this technology opens the door for extensive applications of a RFID system over widespread areas. Mass development of RFID technology has occurred after strong demands for accelerating research and applications. Applications include animal tracking [2], epassports [3], automotive security [4], automated libraries [5], healthcare and pharmaceutical tracking [6], vehicle tolling [7], and access control. The increased breadth of RFID applications has lead to many efforts for creating addtional functionality using RFID systems. Real-time localization is one of the most desired additional features for RFID.

Localization is the process of determining the unknown position of an object based upon measurements gathered in the environment. A real-time localization system (RTLS) is a system that determines the position in real-time [8]. The Global Positioning System (GPS) is perhaps the most famous example of a RTLS. The adoption and application of RTLSs are expected to grow annually for at least the next ten years [9].

For a radio frequency RTLS, whether active or passive, some measurement of the received beacon signals must be made in order to compute an estimate of the target's position. The most common measurements are:

- Time of Arrival (ToA)

- Time Difference of Arrival (TDoA)

- Angle of Arrival (AoA)

- Direction of Arrival (DoA)

- Received Signal Strength Indicator (RSSI)

ToA and TDoA techniques measure signal propagation time and require the clocks of disparate elements in a system to be precisely synchronized. This is very difficult in large scale deployments. Measuring the propagation time of a RF signal requires extremely precise timing synchronization. For example, RF signals travelling at $3 \times 10^{8} \mathrm{~m} / \mathrm{sec}$ require a synchronization accuracy of 3.33 nanoseconds to achieve a distance accuracy of 1 meter. AoA is defined as the angle between the propagation direction of an incident wave and some reference direction, which is known as orientation [10]. DoA indicates both elevation and azimuth angle in a planar array [11].

For RFID systems, the reader interprets and reports the radio frequency messages emitted by the RFID tags. Received Signal Strength Indicators are commonly built into the transceiver chips used in commercial RFID readers and thus this measurement method does not require additional hardware. A RF propagation model is typically used to perform localization using a Received Signal Strength (RSS) measurement.

Here, we investigate the implementation and performance of a received signal strength based RTLS in a commercial RFID system with active tags. The RFID readers each have RSSIs used for ranging. Localization is accomplished using the linear least squares (LSQ) optimization algorithm on the range values. The primary challenge is to develop a robust RSS-based ranging algorithm that is capable of achieving acceptable localization accuracy in indoor environments without additional hardware that would increase the cost of the system. 
This paper is organized as follows. In Section II, we describe related work and the theoretical background for ranging using propagation modeling with RSSI and RFID. Section III presents the details of the localization algorithm and Section IV presents the experimental results and performance. Section $\mathrm{V}$ analyzes improvements in localization accuracy relative to tag orientation issues by exploring tag multiplicity. Section VI presents the current 3D extension research. Finally, in Section VII we conclude the paper and provide insight for future work.

\section{BACKGROUND AND RELATED WORK}

A variety of localization systems based on RFID have been proposed [12]-[20]. The biggest challenge in using RSSI ranging for indoor localization is the difficultly in determining an accurate propagation model. This difficulty arises primarily from multipath propagation. Environments with many reflecting surfaces create multiple transmissions paths between the transmitter and the receiver. At the receiver, the multiple transmission paths result in multiple received signals that can add constructively or destructively which causes the RSSI value, and hence the distance measurement, to vary significantly. In active RFID systems, the transmit power (battery level), tag orientation, reader antenna orientation, and differences in tag characteristics also affect RSSI values. There are two primary methods of RSSI based ranging: RSSI-mapping and propagation modeling.

RSSI-mapping, sometimes referred to as fingerprinting, radio-mapping, and RSS-profiling, was first proposed in the RADAR system [21]. This is the most common RSSI ranging method [22]-[24] and occurs in two phases. The first is the training phase during which numerous RSSI samples are gathered at known locations within the environment to create the RSSI-map. This occurs before any objects are actually localized. The second phase is the estimation phase where real-time RSSI values are converted to distances or directly to a position estimate by comparing them to the RSSI-map. The main problem with RSSI-mapping is that an accurate representation of an environment requires RSSI values to be collected for virtually all tag positions and tag orientations.

The relationships of the power transmitted from tag to reader is expressed by the Friis transmission equation for freespace [25]

$$
P_{R X}=P_{T X}\left[\frac{\sqrt{G_{T X}} \sqrt{G_{R X}} \lambda}{4 \pi d}\right]^{2}
$$

where where $P_{R X}$ and $P_{T X}$ are the received power and transmitted power in watts, respectively, $G_{R X}$ and $G_{T X}$ are the receiver and transmitter antenna gains, respectively, $\lambda$ is the wavelength of the signal in meters, and $d$ is the distance from the transmitter to the receiver in meters.

As noted earlier, propagation modeling is a method for converting RSSI values into a distance measurement by having a propagation model that mathematically characterizes the RSSI value as a function of distance [26]-[28]. The main problem with propagation modeling is the difficulty in determining accurate propagation models in indoor environments with multipath fading. One of the most common approaches taken is to use the log-normal propagation model [29]

$$
P_{R X}[\mathrm{~dB}]=P L\left(d_{0}\right)-10 \eta \log _{10} \frac{d}{d_{0}}+X_{\sigma},
$$

where $P_{R X}$ represents the received power, $P L\left(d_{0}\right)$ is the path loss for a reference distance $d_{0}$ (typically $1.0 \mathrm{~m}$ ), $\eta$ is the path loss exponent, and $X_{\sigma}$ is a Gaussian random variable with zero mean and variance $\sigma^{2}$ that models the random variation of the RSSI value.

To obtain an estimate of the path loss exponent, extensive measurements must be taken in the environment prior to system deployment by recording several RSSI values at known distances. Several attempts have been made to perform on-site calibration of these parameters for specific environments [30], [31], however, the range for these parameters varies widely for system hardware $\left(P L\left(d_{0}\right)\right)$ and environment $(\eta$ and $\sigma)$. In [32], $\eta$ was found to vary between 1.0 and 7.0 and $\sigma$ varied between 2.0 and 14.0. In [33], several techniques are proposed to adaptively estimate the path loss exponent solely based on power measurements and thus eliminate the need to gather extensive RSSI versus distance data. For indoor environments, propagation characteristics can change significantly over time so both RSSI-maps and propagation model parameters must be frequently updated to maintain accuracy.

LANDMARC [34] is a RTLS for RFID systems with active tags. This system uses the RSSI-mapping approach, however, the RSSI-map is created using previously deployed RFID tags with fixed locations and referred to as reference tags. This adaptive approach to RSSI-mapping eliminates much of the training phase. Localization is then accomplished using a $k$-nearest neighbor algorithm. With reference tags placed on a one square meter grid, the LANDMARC paper was able to achieve a localization error of less than 2.0 meters $100 \%$ percent of the time and a localization error of less than or equal to 1.0 meter $50 \%$ of the time.

An improvement to LANDMARC was proposed in [35] by using fewer reference tags placed every five meters in a $25 \mathrm{~m}^{2}$ environment. They then modified the nearest neighbor approach to put a higher emphasis on the reference tag that is closest to the unknown tag. In [17], another improvement to LANDMARC was proposed by using reference tags every two meters and adding the average error range of all neighboring tags with the estimated tag position found using the nearest neighbor approach.

We propose a new RFID RTLS with a RSSI ranging method using multiple propagation models. The proposed system uses reference tags to perform adaptive propagation modeling which eliminates the need for predetermined propagation model parameters such as the path-loss coefficient. The issue of the tag orientation at the object to be localized is something that is not specifically addressed with the LANDMARC or other RSSI RTLSs. The proposed system also addresses tag orientation. 


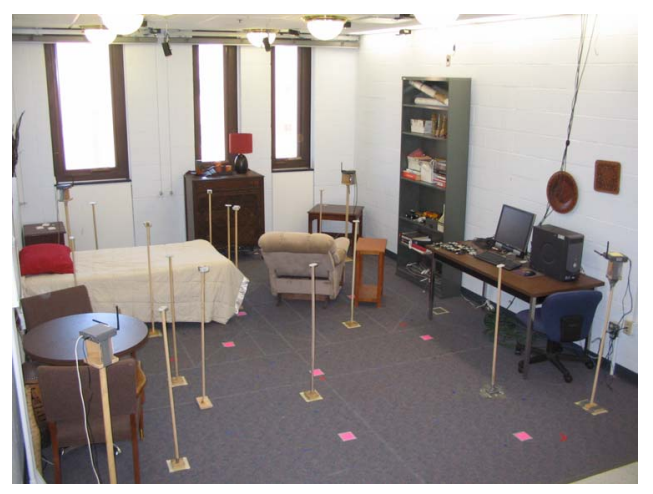

Fig. 1. Photograph of the room used for experimental evaluation of the RFID RTLS.

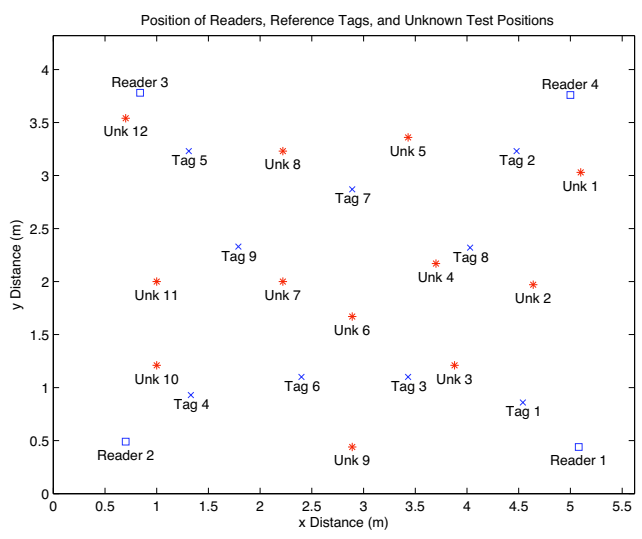

Fig. 2. Deployment schematics showing reader, reference tag and unknown locations.

\section{A NEW RFID RTLS}

The proposed experimental system consists of four RF Code [36] RFID readers in an indoor room environment as shown in Figure 1. The RF Code system operates at $303 \mathrm{MHz}$ and the hardware consists of readers and active tags. The lab room walls are concrete and the floor is covered by carpet. The room environment includes a bed, multiple tables and chairs, and a tall metal shelf that provide many opportunities for multipath propagation. The readers are placed in the corners of the rooms at a height of 1.1 meters with no objects or reflecting surfaces within $60 \mathrm{~cm}$ in any direction. There are 9 total reference tags for the 2D deployment area. All reference tags and unknown tags are placed at a height of 1.0 meter. and each of the reference tags has a line-of-sight (LOS) to each reader. The reference tags are placed so that each reader has a reference tag at distances of approximately 1.0, 2.0, 3.0, 4.0, and 4.5 meters. The localization accuracy of the system is evaluated at 12 different unknown positions (all located within the room dimensions) as shown in Figure 2.

Each reader reports the RSSI value of a tag for each of the reader antennas. Each reader has two antennas.The RSSI values from each of the two reader antennas are averaged over 15 collected samples. The maximum RSSI value between each

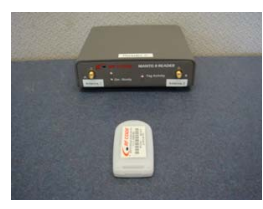

(a) Group 1

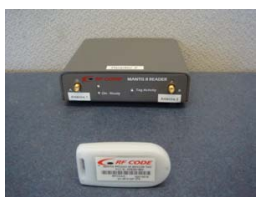

(b) Group 2

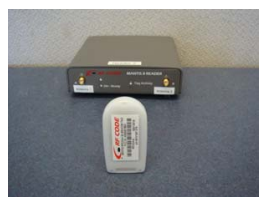

(c) Group 3
Fig. 3. Photographs of example tag group orientations.

antenna reader is selected and reported as the single RSSI value for that reader. The sum of both the RSSI values between each reader antenna is also recorded and used for subarea determination mentioned later.

\section{A. Experimental Methodology}

Prior to system deployment, several reader antenna orientations and tag orientations were compared to find an optimal reader antenna orientation. The five different reader antenna orientations include different combinations of antennas pointing to the ceiling or walls. We investigated 12 tag orientations with each of the 5 reader antenna configurations to find the configuration that had the least variability over all tag orientations. Next, several tags were tested in a simple RSSI vs. distance experiment to make sure that the tags being used in the system deployment had similar RSSI characteristics.

In the actual deployment, each reader sees a given tag with a different orientation. Therefore, we classified 12 key tag orientations into groups by rotation about the z-axis. Group tag orientation 1 is the group of tags having the RF Code label facing toward the ceiling as shown in Figure 3(a), group tag orientation 2 has the RF Code label facing the walls with the text being horizontal as shown in Figure 3(b), and group tag orientation 3 is any tag having the RF Code label facing the walls with the text being vertically positioned as shown in Figure 3(c).

Localization data was collected from each unknown location for 5 different tag orientations within each tag orientation group above. A second set of data was collected on a different day at a different time to capture possible time and interference variations. Thus for each of the 12 unknown positions, a total of 30 data sets were gathered for different orientations and times.

\section{B. Ranging and Localization Algorithm}

Once the RSSI data is collected, it is sent to the localization software to estimate the position of the unknown tag. The algorithm is broken up into the following major steps: (1) subarea detection; (2) propagation modeling; (3) elimination rounds; (4) range averaging; and (5) position estimation.

Step 1: Subarea Detection: The purpose of this step is to attempt to identify the most likely subarea of the room within which the unknown tag is located. Based on the RSSI values from an unknown tag, the quadrant of the room is found which is most likely to contain the coordinates for the unknown tag position by comparing the RSSI values between all the different readers. The quadrant shown in Figure 4, is 


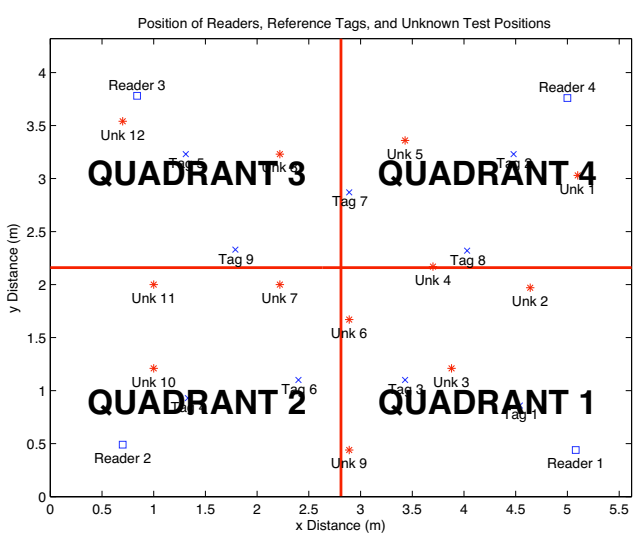

Fig. 4. Schematic illustrating quadrant detection.

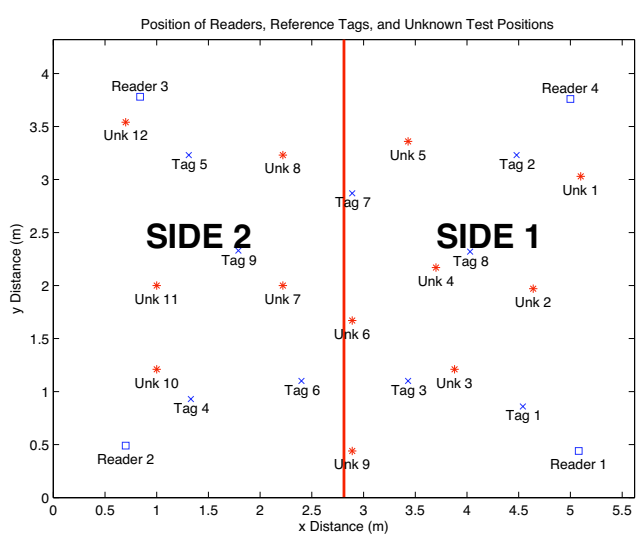

Fig. 5. Schematic illustrating horizontal side of room detection.

the area where the ranges and coordinates for the unknown tag are expected to be. Range estimates for an unknown tag are considered most accurate within the determined quadrant.

For the unknown tag, the sum of the RSSI values on each antenna is found for each reporting reader. Whichever reader reports the maximum RSSI sum is chosen as the most likely quadrant. The reader with the second largest RSSI sum is also recorded at this time as the "backup" quadrant for possible use later in the algorithm.

Step 2: Propagation Modeling: Based on the previously determined quadrant, two propagation models are created by using the reference tags in the two adjacent sides of the room shown in Figures 5 and 6. For example, if Quadrant 1 is chosen in step 1, then the adjacent sides to that quadrant are Side 1 and Side 4. Similarly, if we are in Quadrant 3, the adjacent sides to that quadrant are Side 2 and Side 3.

$$
\begin{aligned}
\text { Reference Tags on Side } 1 & =\{1,2,3,7,8\} \\
\text { Reference Tags on Side } 2 & =\{4,5,6,7,9\} \\
\text { Reference Tags on Side } 3 & =\{2,5,7,8,9\} \\
\text { Reference Tags on Side } 4 & =\{1,3,4,6\}
\end{aligned}
$$

After the reference tags are chosen for each model, a linear

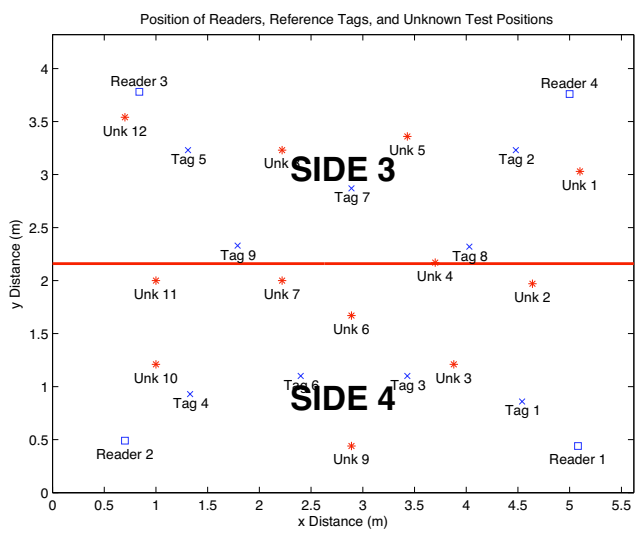

Fig. 6. Schematic of vertical side of room detection

model is fit to each set of data points using their actual known distances and the maximum RSSI value obtained from that reader. Linear models were experimentally shown to be good approximations for propagation modeling for this size of deployment. The linear model fitting is done for RSSI values in $\mathrm{dB}$. An additional data point is added for each reader at a distance of 0.0 meters and with an RSSI value equal to the maximum power that a reader can obtain,- $40.0 \mathrm{~dB}$.

After the propagation models are created for each reader, the range estimates are found for a target for each reader by solving the linear models using the maximum RSSI value received from that target.

During experimentation it was determined that for tags in close proximity to a reader, a third model could be generated to more accurately fit that data. This "close proximity" model is created using the data point $(0.0,-40.0)$, the reference tag that is closest to the reader, and then one other reference tag which yields the largest linear slope. For example, consider the propagation models created for Reader 4 from Unknown 1 as shown in Figure 7. Specifically, "Unknown 1" represents a collection of RSSI values for a tag that has unknown location information. For experimental purposes, the tag location is known and it is shown in Figure 7 to illustrate its actual RSSI values and its actual location. The determined side of the room is Side 1, so Model 1 is created using Reference Tags 1, 2, 3, 7 , and 8 . The determined quadrant is Quadrant 4 , so the second adjacent side is Side 3. Therefore, Model 2 is created using Reference Tags 2, 5, 7, 8, and 9. For the propagation models created for each reader, the range estimates are found for a target for each reader by solving the linear models using the maximum RSSI value received from that target. Finally, the third model is found by using three data points. The maximum RSSI value and distance 0 meters, the closest reference tag (Reference Tag 2), and the third data point is the result of finding the maximum slope between all other reference tags. For this reader, using Reference Tag 7 yields the linear model with the maximum slope.

Step 3: Elimination Rounds: All of the estimated ranges are put through a series of elimination rounds to remove any 


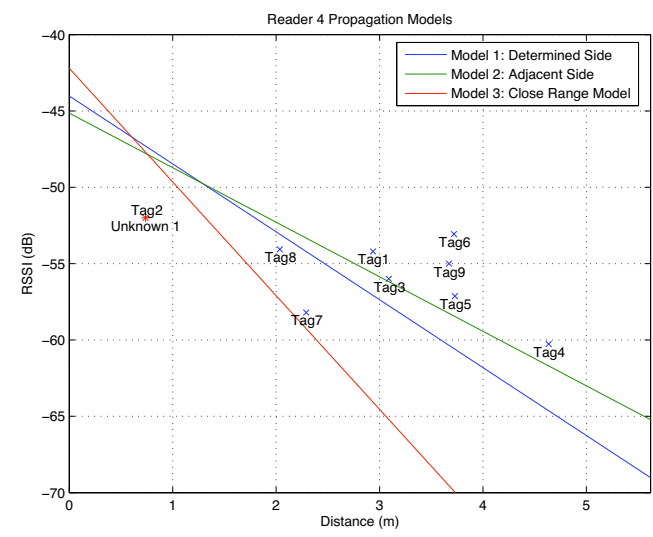

Fig. 7. Propagation models created from Reader 4 for Unknown 1.

obvious errors due to inaccurate propagation modeling.

Elimination 1: Out of Room Ranges The first round of eliminations simply removes any ranges that would be outside of the room dimensions from a reader. This is due to the fact that all unknowns are deployed with the assumption that they are within the room dimensions.

Elimination 2: Proximity The second round of eliminations is based on close proximity to readers. Each reader has a predetermined RSSI threshold value for close proximity. If this RSSI threshold is exceeded, then a tag is determined to be "close" to a reader and all other ranges must fall within a specified radius from that reader to be considered valid. If an unknown tag is determined to have close proximity to a reader and other reader ranges do not indicate so, those ranges are eliminated.

For example, Figure 8 shows the case for Unknown 10. The two lines are the first range and the second range obtained from the propagation modeling step. The maximum RSSI value for that position exceeds the RSSI threshold for that reader so we indicate a close proximity to that reader. For this case, we eliminate the first range from Reader 1 and the second range from Reader 2 because they are both outside the proximity threshold.

The converse is also checked. That is, if a range indicates that an unknown would be in close proximity to a reader and the RSSI threshold is not met, then this range must also be eliminated.

For example, Figure 9 shows the case for Unknown 5. No reader has been labeled in close proximity, however, the second range from Reader 3 indicates that the unknown position would have to be in close proximity to Reader 1 by the range only lying in the corner of the room. Hence, the second range from Reader 3 is eliminated.

Elimination 3: Quadrant Elimination

Finally, based on the determined quadrant, any ranges not within this quadrant are also eliminated. For the reader that is in the chosen quadrant, the ranges from that reader are first checked to see if they actually fall within that quadrant. If not, then further tests are performed since the algorithm assumes

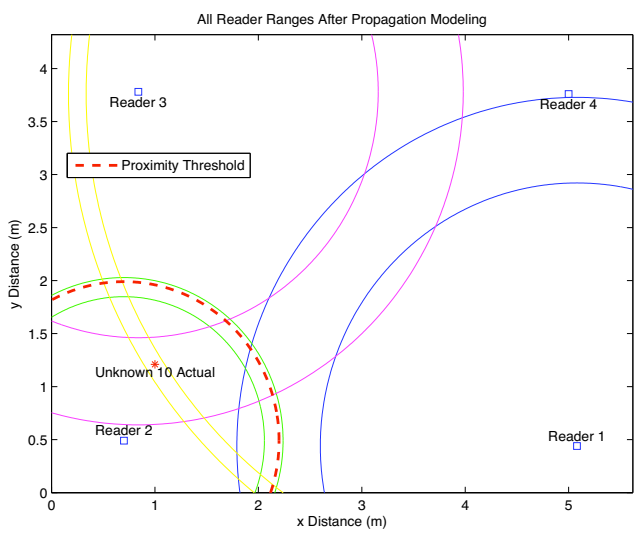

Fig. 8. Unknown 10: All reader ranges with close proximity to Reader 2.

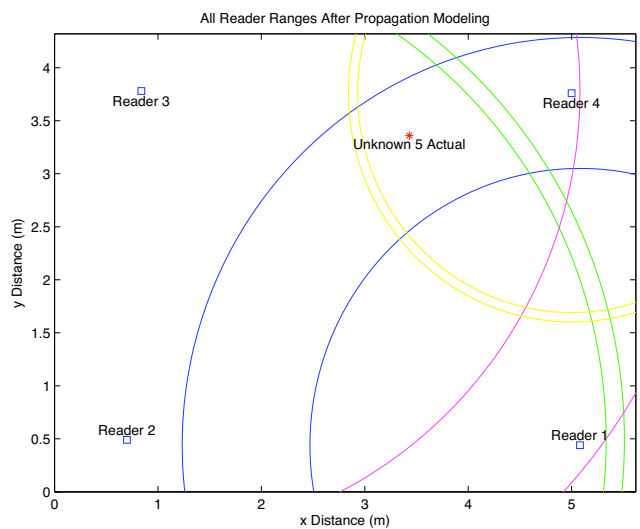

Fig. 9. Unknown 5: All reader ranges with no close proximity.

that the range value from the reader in same quadrant is the most accurate. For example, if the unknown is determined to be in Quadrant 1, and both Reader 1 ranges are outside of the quadrant, then the RSSI value does not fit the models accurately.

When this occurs, the ranges from the other readers are checked to see if they also indicate that the unknown is within the determined quadrant. If they do, the chosen quadrant is assumed to be correct and the propagation model is switched to the close proximity model for the reader in the quadrant. If the other readers indicate the chosen quadrant is not correct, then we switch to the back up quadrant recorded in Step 1.

Referring back to the example of an unknown determined in Quadrant 1, assume the ranges for Reader 1 are both outside of the chosen quadrant. We then look at the ranges from readers 2,3 , and 4 . If the ranges from all of the readers are located within Quadrant 1, it is determined that the quadrant is accurate, but the model for Reader 1 is not, so the reader will switch to the close proximity model. If the ranges from all the readers were not located in Quadrant 1, then it is determined that the chosen quadrant is not accurate and a switch is performed to the backup quadrant. 
Step 4: Range Averaging: If both ranges from any reader have successfully made it through all the elimination rounds, then they are averaged together to create a single range from each reader. If all four readers have similar RSSI values, then the unknown is assumed to be in the middle of the room which facilitates the filtering of intersection points and LSQ estimation. In the experiments, similar meant that the readers had RSSI values within $2 \mathrm{~dB}$ of each other. If this criteria is met, then all the original ranges are used for averaging.

Step 5: Position Estimation: It was discovered through initial experiments that the reader which lies in the chosen quadrant frequently has more accurate range estimates than the other readers. Performing LSQ with one accurate range and three very inaccurate ranges results in significant degradation in the localization accuracy. In an effort to improve the localization accuracy, it is desirable to emphasize the accurate range value. In order to do this, the algorithm takes into consideration the intersection points, if they exist, between the range from the closest reader and the ranges from all remaining readers.

\section{Intersection Points}

This idea comes from the theory of trilateration. Given three readers with perfect ranging, all three would intersect at one point indicating the position of the target. With imperfect ranging, the ranges do not intersect in a single point, but their points of intersection can give some insight to the location of the target. Imperfect ranges from two readers will intersect in zero, one or two points. If there are no intersection points, there may be a "point of interest" where the ranges from two readers come close to each other but do not intersect. If this occurs within a difference threshold of 0.4 meters, the point of interest is chosen to be the midpoint between these two ranges.

\section{LSQ Estimates}

After the ranges have been averaged, all possible combinations of the remaining reader ranges are used to calculate several LSQ estimates of the position. The intersection points and LSQ estimates are first filtered to make sure they are on the correct quadrant, or if they are in the middle of the room, or if they are in proximity to a reader previously determined to be close. After the filter round, all valid intersection points and LSQ estimates are averaged together to find a single position estimate.

A schematic illustrating this process is shown in Figure 10 for an unknown. In Figure 10, various colored stars are either the true location or estimations of that unknown by different methods. The unknown was determined to be in Quadrant 1, so the range from Reader 1 is emphasized. The corresponding intersection points are found between Reader 1 and all other readers and indicated with a green asterisk. Note that Reader 3 and Reader 1 do not intersect, so the point of interest is the point of minimal distance between the ranges. This intersection point is within the 0.4 meter threshold of the Reader 1 range and therefore will contribute to averaging. The LSQ estimates are found using all possible combinations of the three ranges and are indicated with a magenta asterisk. Intersection points

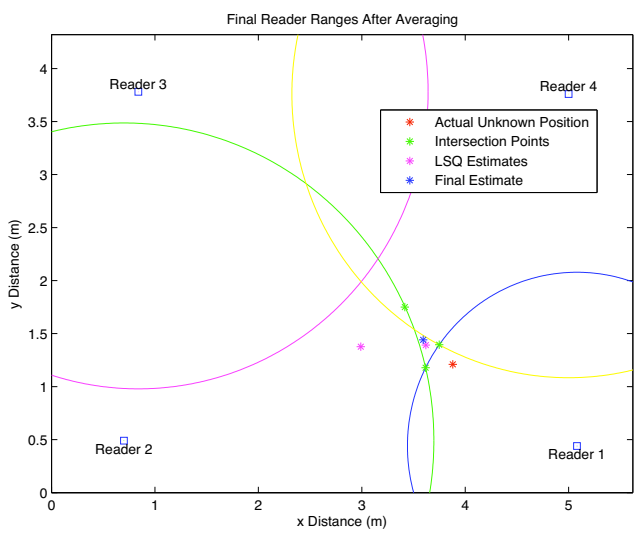

Fig. 10. Intersection points, and LSQ estimates for Unknown 3, representing by colored stars.

and LSQ estimates are first filtered to only use the points that are within the determined quadrant and those that remain are averaged to find the final position estimate indicated by the blue asterisk.

\section{EXPerimental Results ANd PERformance}

Once again, previous studies suggest LANDMARC is able to achieve localization errors of less than 2.0 meters $100 \%$ percent of the time and a localization error of less than or equal to 1.0 meter $50 \%$ of the time. These results are adopted as baseline performance metric for the proposed algorithm.

The algorithm was first tested with the optimal tag orientation (group tag orientation 1) in which the unknown tags belong to the same orientation group as the reference tags. The results are shown in Table I where the Individual column represents each different tag orientation and position combination as a unique position and the Average for Unknown is the performance averaged over all the tag orientations for the specific unknown position.

TABLE I

LOCALIZATION RESUlts: 4 READERS With OPTIMAL TAG ORIENTATION

\begin{tabular}{|l|c|c|c|}
\hline \hline Error (meters) & Goal & Individual & Average for Unknown \\
\hline$E \leq 1.0$ & $50.0000 \%$ & $57.50 \%$ & $58.33 \%$ \\
$1.0<E \leq 1.5$ & n/a & $23.33 \%$ & $25.00 \%$ \\
$1.5<E \leq 2.0$ & n/a & $10.83 \%$ & $8.33 \%$ \\
$2.0<E$ & $0.0000 \%$ & $8.33 \%$ & $8.33 \%$ \\
\hline \hline
\end{tabular}

As shown in the table, a localization error less than or equal to 1.0 meter was obtained $58.33 \%$ of the time, which exceed the performance of the LANDMARC system. However, the proposed system also has localization errors greater than 2.0 meters which is worse than the LANDMARC system. A detailed analysis of the experimental data revealed that Unknown 9 is the single contributor to these high localization errors. The difficulty with Unknown 9 is that its signal suffers more reflections in the environment than the other tags because 
it is located within $45 \mathrm{~cm}$ of the wall and there is a set of table and chairs nearby.

Further analyzing the results from Unknown 9 reveals that it also has the most variability as a function of tag orientation. Even within the optimal group tag orientation, small changes in the tag orientation about the $\mathrm{z}$-axis have a significant effect on the RSSI values obtained by Readers 1 and 2 . This significantly degrades the overall localization accuracy performance. In order for an RTLS to be considered viable, it must be robust to all tag orientations. With this in mind, we consider adding a second tag of different orientation to the unknown position.

\section{ImPROVEMENTS USING TAG MULTIPLICITY}

One way to exploit diversity in an RFID system is to include additional readers. However, since the readers are the key contributors to system cost, this is not the most desirable approach. Another way to exploit diversity is by using tag multiplicity, i.e., deploying multiple tags at a single position [37].

The algorithm described in Section III relies on the maximum RSSI value between antennas of a reader. Occasionally both of these antenna values may indicate that an unknown tag is farther away than it actually is (as previously described for Unknown 9 with Readers 1 and 2). By deploying a second tag at the unknown position with a slightly different orientation, but within the same tag orientation group, it is possible that a reader may receive a "weak" RSSI value from one tag, but a "strong" RSSI value from the second tag. The experiments in Section III were repeated with a second tag deployed at each of the unknown positions. The second tag has an orientation of the same group as the first, but rotated 180 degrees about the z-axis. The results from this experiment are shown in Table II.

TABLE II

LOCALIZATION RESUlTS: 4 READERS With OPTIMAL TAG ORIENTATION AND TAG MULTIPLICITY

\begin{tabular}{|l|c|c|c|}
\hline Error (meters) & Goal & Individual & Average for Unknown \\
\hline$E \leq 1.0$ & $50.00 \%$ & $58.33 \%$ & $58.33 \%$ \\
$1.0<E \leq 1.5$ & n/a & $30.83 \%$ & $25.00 \%$ \\
$1.5<E \leq 2.0$ & n/a & $9.17 \%$ & $16.67 \%$ \\
$2.0<E$ & $0.00 \%$ & $1.67 \%$ & $0.00 \%$ \\
\hline \hline
\end{tabular}

The use of tag multiplicity has improved the overall performance of the system. A localization error of less than or equal to 1.0 meter is still achieved $58.33 \%$ of the time, while localization errors greater than 2.0 meters are eliminated. The performance improves for all combinations of tag position and orientation as indicated by the Individual column of Table II with only very small percentage of localization errors of greater than 2.0 meters.

The experiment was also repeated for unknown positions belonging to tag orientation groups 2 and 3. Table III shows the localization performance for the three tag orientation groups using the average error for each unknown position and Table IV shows the overall system performance for the optimal tag orientation and all tag orientations. Using tag multiplicity for all orientations showed that the performance goal was obtainable for tag orientations within groups 1 and 3 . Tag orientation group 2 , however, showed a significant performance decrease for localization error of less than or equal to 1.00 meter while the performance goal was still obtained for localization error of greater than 2.00 meters.

TABLE III

Localization Results: 4 ReAders with TAg Multiplicity for All ORIENTATIONS

\begin{tabular}{|l|c|c|c|c|}
\hline \multirow{2}{*}{ Error (meters) } & \multirow{2}{*}{ Goal } & \multicolumn{3}{|c|}{ Tag Orientation } \\
\cline { 3 - 5 } & & Group 1 & Group 2 & Group 3 \\
\hline$E \leq 1.0$ & $50.00 \%$ & $58.33 \%$ & $33.33 \%$ & $50.00 \%$ \\
$1.0<E \leq 1.5$ & $\mathrm{n} / \mathrm{a}$ & $25.00 \%$ & $25.00 \%$ & $41.67 \%$ \\
$1.5<E \leq 2.0$ & $\mathrm{n} / \mathrm{a}$ & $16.67 \%$ & $41.67 \%$ & $8.33 \%$ \\
$2.0<E$ & $0.00 \%$ & $0.00 \%$ & $0.00 \%$ & $0.00 \%$ \\
\hline \hline
\end{tabular}

TABLE IV

Localization Results: 4 READERs With TAG Multiplicity

\begin{tabular}{|l|c|c|c|}
\hline Error (meters) & Goal & Optimal Orientation & All Orientations \\
\hline$E \leq 1.0$ & $50.0000 \%$ & $58.3333 \%$ & $47.2222 \%$ \\
$1.0<E \leq 1.5$ & n/a & $25.0000 \%$ & $30.5556 \%$ \\
$1.5<E \leq 2.0$ & n/a & $16.6667 \%$ & $22.2222 \%$ \\
$2.0<E$ & $0.0000 \%$ & $0.0000 \%$ & $0.0000 \%$ \\
\hline
\end{tabular}

\section{EXtension to 3D SPACE}

The proposed model was also preliminarily extended to $3 \mathrm{D}$ space with 4 readers and 24 reference tags implemented. The concept of subarea detection of unknown tag in the 2D model was inherited. While in the 3D model, it was checked on the $\mathrm{x}, \mathrm{y}$, and $\mathrm{z}$ planes at the same time. Comparing to 2D model's 2 by 2 quadrant detection, the current 3D model was designed to perform in a 4 by 3 by 4 cell detection.

The exploratory experiment was conducted with tag multiplicity. The unknown tags were distributed within the room at 8 different locations with 3 different orientations for each location. The average performance is summarized in Table V.

TABLE V

\begin{tabular}{|c|c|c|}
\hline Error (meters) & Individual & Average \\
\hline$E \leq 1.0$ & $29.17 \%$ & $8.33 \%$ \\
\hline $1.0<E \leq 1.5$ & $15.28 \%$ & $20.83 \%$ \\
\hline $1.5<E \leq 2.0$ & $25.00 \%$ & $50.00 \%$ \\
\hline $2.0<E \leq 2.5$ & $9.72 \%$ & $20.83 \%$ \\
\hline $2.5<E \leq 3.0$ & $11.11 \%$ & $0.000 \%$ \\
\hline $3.0<E$ & $9.72 \%$ & $0.000 \%$ \\
\hline
\end{tabular}

When tags are treated individually, a localization error of less than or equal to 2.0 meters is achieved $69.44 \%$ of the 
time. The use of tag multiplicity has improved the overall performance of the system. A localization error of less than or equal to 2.0 meter is achieved $79.17 \%$ of the time, while localization errors greater than 2.5 meters are eliminated.

\section{CONClusions And Future Work}

This paper presented a RSSI based RTLS for use with an active RFID system. Reference tags and multiple propagation models are proposed and used to improve the performance of RSSI based ranging. The advantages of the proposed system over other existing systems are listed here. First, no modifications of existing hardware devices is required, therefore, further decreasing the system complexities. Second, the system is robust enough to adapt to different environments. By using reference tags to adaptively create propagation models, there is no need to measure propagation model parameters such as the path loss exponent. Third, the system uses significantly fewer reference tags than the LANDMARC system.

Future work will be conducted in a variety of ways to promote a RTLS system with better performance on characteristics of scalability, robustness and accuracy. Besides tag multiplicity for the unknown tags, this multiplicity characteristic is expected to continue improving the accuracy of the system by cooperating with reference tags. The scalability of the current multiple linear propagation models will be investigated using in-depth analysis on a large scale experiment environment.

\section{REFERENCES}

[1] Harvey Lehpamer, "RFID Design Principles," Artech House, Inc., 2008.

[2] (2005) RFID Used in Advanced Cattle Tracking System. [Online]. Available: http://www.rfidupdate.com/articles/index.php?id=754

[3] (2006) Us Government Issues 300 RFID Passports. [Online]. Available: http://www.rfidupdate.com/articles/index.php?id $=1073$

[4] (2006) RFID Drives into Auto Manufacturing. [Online]. Available: http://www.rfidupdate.com/articles/index.php?id=1078

[5] J. Burnell. (2008) Large Swiss Library System Implementing RFID. [Online]. Available: http://www.rfidupdate.com/articles/index.php?id=1532

[6] A. Cangialosi, J. Monaly, and S. Yang, "Leveraging RFID in Hospitals: Patient Life Cycle and Mobility Perspectives," IEEE Communications Magazine, vol. 45, no. 9, pp. 18-23, September 2007.

[7] (2007) Sirit Lands 2m RFID Contract for California Toll Roads. [Online]. Available: http://www.rfidupdate.com/articles/index.php?id=1331

[8] D. P. Harrop, G. Holland, and R. Da, "Real Time Locating Systems 2008-2018," IDTechEx, Tech. Rep., 2008.

[9] J. Burnell, "Innovation to Drive Strong RTLS Adoption," RFID Update, vol. 889, August 132008.

[10] R. Peng and M. L. Sichitiu "Angle of Arrival Localization for Wireless Sensor Networks," In Proceedings of IEEE SECON, pp: 374-382, Sep. 2006.

[11] [Online]. Available: http://www.edaboard.com/thread173060.html

[12] D. Hahnel, W. Burgard, D. Fox, K. Fishkin, and M. Philipose, "Mapping and Localization with RFID Technology," In IEEE Proceedings of Internationl Conference on Robotics and Automatin (ICRA), vol.1, pp: 1015-1020, 2004.

[13] B. Xu and W. Gang, "Random Sampling Algorithm in RFID Indoor Location System," In Third IEEE International Workshop on Electronic Design, Test and Aplications (DELTA), pp. 168-176, 2006.

[14] Radio Frequency Identification (RFID) home page [Online]. Available: http://www.aimglobal.org/technologies/rfid/

[15] D. Joho, C. Plagemann, and W. Burgard, "Modeling RFID Signal Strength and Tag Detection for Localization and Mapping," Proceedings of IEEE International Conference on Robotics and Automation (ICRA), May 2009, pp.3160-3165.
[16] J. S. Choi, H. Lee, R. Elmasri and D. W. Engels, "Localization Systems Using Passive UHF RFID," Fifth International Joint Conference on INC, IMS and IDC,2009, pp.1727-1732.

[17] G. Jin, X. Lu, and M. S. Park, "An Indoor Localization Mechanism Using Active RFID Tag," IEEE International Conference on Sensor Networks, Ubiquitous, and Trustworthy Computing, vol. 1, pp. 40-43, 05-07 June 2006

[18] C. Huang,P. Chung, M. Tsai, Y. Yang and Y. Hsu, "Reliability Improvement for an RFID-based Psychiatric Patient Localization System," Computer Communications, Vol. 31, Issue 10, 2008, pp.2039-2048.

[19] D. Hahnel, W. Burgard, D. Fox, K. Fishkin and M. Philipose, "Mapping and Localization with RFID Technology," Preceedings of IEEE International Conference on Robotics and Automation, Vol. 1, 26. May 2004, pp.1015-1020.

[20] A. Chattopadhyay, and A. R. Harish, "Analysis of Low Range Indoor Location Tracking Techniques using Passive UHF RFID Tags," IEEE Radio and Wireless Symposium, 22-24 Jan. 2008, pp.351-354.

[21] P. Bahl and V. Padmanabhan, "Radar: An In-building RF-based user Location and Tracking System," In IEEE Proceedings of Nineteenth Annual Joint Conference on Computer and Communications Societies (INFOCOM), vol. 2, pp. 775-784 vol.2, 2000.

[22] C. Rohrig and F. Kunemund, "Estimation of Position and Orientation of Mobile Systems in a Wireless LAN," 46th IEEE Conference on Decision and Control, pp. 4932-4937, 12-14 Dec. 2007.

[23] J. Ralston, C. Hargrave, and D. Hainsworth, "Localisation of Mobile Underground Mining Equipment Using Wireless Ethernet," Industry Applications Conference. Fourtieth IAS Annual Meeting, vol. 1, pp. 225230 Vol. 1, 2-6 Oct. 2005.

[24] C. Alippi, A. Mottarella, and G. Vanini, "A RF Map-based Localization Algorithm for Indoor Environments," IEEE International Symposium on Circuits and Systems, pp. 652-655 Vol. 1, 23-26 May 2005.

[25] J. L. Brchan, "Indoor Localization in An Active RFID System with Multiple Propagation Models," Master Thesis, University of NebraskaLincoln, Aug 2009.

[26] S. Zhou and J. Pollard, "Position Measurement Using Bluetooth," IEEE Transactions on Consumer Electronics, vol. 52, no. 2, pp. 555-558, May 2006.

[27] Y. Chen and H. Kobayashi, "Signal Strength Based Indoor Geolocation," IEEE International Conference on Communications, vol. 1, pp. 436-439, 2002.

[28] R. Akl, D. Tummala, and X. Li, "Indoor Propagation Modelinlg at 2.4 GHZ for IEEE 802.11 Networks," Wireless Networks and Emerging Technologies, July 2006.

[29] T. Rappaport, "Wireless Communications: Principles and Practice, ," 2e. Prentice Hall, 2002.

[30] S. Ghassemzadeh, L. Greenstein, A. Kavcic, T. Sveinsson, and V. Tarokh, "Uwb Indoor Path Loss Model for Residential and Commercial Buildings," 58th IEEE Vehicular Technology Conference, vol. 5, pp. 3115-3119 Vol.5, Oct. 6-9 2003.

[31] K. Yedavalli, B. Krishnamachari, S. Ravula, and B. Srinivasan, "Ecolocation: a Sequence Based Technique for RF Localization in Wireless Sensor Networks," Fourth International Symposium on Information Processing in Sensor Networks, pp. 285-292, April 152005.

[32] H. Hashemi, "The Indoor Radio Propagation Channel," Proceedings of the IEEE, vol. 81, no. 7, pp. 943-968, Jul 1993.

[33] G. Mao, B. D. O. Anderson, and B. Fidan, "Path Loss Exponent Estimation for Wireless Sensor Network Localization," Computrt Networks, vol. 51, no. 10, pp. 2467-2483, 2007.

[34] L. Ni, Y. Liu, Y. C. Lau, and A. Patil, "Landmarc: Indoor Location Sensing Using Active RFID," Proceedings of the First IEEE International Conference on Pervasive Computing and Communications (PerCom), pp. 407-415, 23-26 March 2003.

[35] S. T. Shih, K. Hsieh, and P. Y. Chen, "An Improvement Approach of Indoor Location Sensing using Active RFID," First International Conference on Innovative Computing, Information and Control (ICICIC), vol. 2, pp. 453-456, 30-01 Aug. 2006.

[36] Rf code, inc. [Online]. Available: http://www.rfcode.com/

[37] N. Vaidya and S. R. Das, "RFID-based Networks - Exploiting Diversity and Redundancy," WINGS Lab Technical Report, June 2006. 